\title{
Short stature as an independent risk factor for cephalopelvic disproportion in a country of relatively small-sized mothers
}

\author{
Rusleena Toh-adam • Kasemsri Srisupundit • \\ Theera Tongsong
}

Received: 14 August 2011 / Accepted: 28 November 2011 / Published online: 21 December 2011

(C) The Author(s) 2011. This article is published with open access at Springerlink.com

\begin{abstract}
Objective To clarify the relationship between maternal height and cesarean rate due to cephalopelvic disproportion (CPD) in singleton pregnancies among ethnic groups of relatively short stature.

Methods A retrospective cohort study was performed on Thai singleton pregnancies at gestational age of more than 34 weeks. Logistic regression analysis was performed to correlate the maternal height and a risk for CPD. The short stature was defined by a cut-off value at 5 th percentile ranking. Odds ratio for CPD was determined.

Results Of 11,026 recruited, 9,198 were available for analysis. Considering cut-off value of $145 \mathrm{~cm}$, short stature was significantly associated with higher rate of CPD with odds ratio of $2.4(95 \%$ CI 1.8-3.0). The odds $=\exp (4.048-0.042 \times \mathrm{Ht})$. After control of other variables, the relationship between maternal height and rate of CPD was still high.

Conclusion Mothers with short stature were significantly correlated with a higher rate of CPD, even after control of birth weight, parity and type of attendance. Clinical points could be drawn from this study including (1) definition of short statue must be developed for particular geographic or ethnic groups. In Thai population, using $145 \mathrm{~cm}$ as a cut-off value, odds of CPD is 2.4; (2) Probability of CPD may be estimated by maternal height as a single variable or multiple variables using logistic regression equations.
\end{abstract}

Keywords Cephalopelvic disproportion - Cesarean section · Maternal height · Short stature

R. Toh-adam $\cdot$ K. Srisupundit $\cdot$ T. Tongsong $(\bowtie)$

Department of Obstetrics and Gynecology,

Faculty of Medicine, Chiang Mai University,

Chiang Mai, Thailand

e-mail: ttongson@mail.med.cmu.ac.th

\section{Introduction}

Maternal morbidity and mortality remain a major challenge to health systems worldwide, especially in developing countries. Recent estimates indicate that more than 300,000 women die worldwide in 2008 [1]. In developing countries, a significant number of maternal deaths are attributable to the complications of obstructed labor leading to birth canal trauma, postpartum hemorrhage, and genital infections, etc. Of patients with dystocia, delay in the decision to seek care or delay in arrival to an appropriate medical care facility is common in rural hospitals. Detection of mothers at a higher risk of dystocia is therefore important for higher precaution, close attention or preparation of cesarean section or timely labor induction in daytime when more manpower and experienced physicians are available. This is particularly crucial in settings where cesarean section is not feasible, so that women at high risk for CPD may be referred to a hospital equipped with an operating theatre before the onset of labor. Maternal height reflecting maternal pelvic size, which is related to dystocia, has been shown to be predictive for obstructed labor [2-8]. Indeed, maternal height, as a simple tool requiring no any special skills, has been investigated extensively especially in developing countries for predicting obstructed labor. However, whereas the inverse relationship between maternal height and the cesarean rate has been consistently demonstrated, the application of height to predict risk of obstructed labor or cesarean section remains controversial. Furthermore, no universal definition of short stature in pregnant women has been well accepted. This is due to the fact that maternal height distribution is varied among different geographic or ethnic groups of study. Thai or Southeast Asian mothers are typically shorter than those in western. Certainly, application of maternal height on clinical practice based on studies in 
other parts of the world is inappropriate. Therefore, this study aimed to determine the effectiveness of maternal height in predicting risk of cesarean section due to cephalopelvic disproportion among Thai population.

\section{Patients and methods}

A retrospective cohort study was conducted at Maharaj Nakorn Chiang Mai Hospital, Chiang Mai University, Thailand during 2006-2010. This study was ethically approved by the local institutional review board. The database of maternal-fetal medicine unit and some medical records was reviewed for baseline characteristics, maternal height and labor outcomes. Some missing data, especially the maternal height, were traced from the patients either by mail, phone or direct search of census registration from Department of Provincial Administration. Details of delivery were prospectively recorded by the obstetricians and computerized collected everyday by the research supporting nurse. Inclusion criteria were (1) gestational age of greater than 34 weeks based on overall clinical estimation of medical records, (2) singleton pregnancies, and (3) available data of delivery method with indication of cephalopelvic disproportion (CPD). In our practice, CPD or the failure to progression of labor is defined as suggested by the Royal Thai College of Obstetricians and Gynecologists [9], as follows: (1) active phase of labor defined by cervical dilatation of at least $4 \mathrm{~cm}$ and $80 \%$ effacement, (2) regular and adequate uterine contractions at least $2 \mathrm{~h}$ before decision making, and (3) abnormal partograph, such as protraction disorders, arrest disorders or second stage failure to descend. Variables included for analysis were maternal height, maternal age, parity, type of attendance (private or general antenatal care), birth weight and delivery methods. The pregnant women were categorized into several subgroups based on percentile ranking of maternal height distribution, parity, and type of attendance. The main outcome was cesarean section rate indicated by CPD in various subgroups of maternal height.

Table 1 Cesarean section rate and odds ratio according to categorized groups

\begin{tabular}{llll}
\hline & No. of CPD & CPD & Odds ratio (95\% CI) \\
\hline Height $\leq 145 \mathrm{~cm}$ & $402(83.8 \%)$ & $78(16.3 \%)$ & $2.353(1.824-3.037)$ \\
Height $\leq 150 \mathrm{~cm}$ & $1803(88.6 \%)$ & $233(11.4 \%)$ & $1.689(1.434-1.989)$ \\
Height $\leq 155 \mathrm{~cm}$ & $4336(90.4 \%)$ & $460(9.6 \%)$ & $1.550(1.328-1.808)$ \\
Private cases & $1257(86.4 \%)$ & $198(13.6 \%)$ & $2.085(1.753-2.479)$ \\
Nulliparity & $4568(87.8 \%)$ & $633(12.2 \%)$ & $4.943(4.016-6.084)$ \\
\hline
\end{tabular}

Statistical analysis

Means and standard deviation for different variables in each group were calculated and tested for significance. Odd ratios with $95 \%$ confidence intervals for cesarean rate due to CPD in various groups of maternal height were determined. $p<0.05$ was considered statistically significant. A logistic regression analysis was performed to identify if there was a particular height as well as other variables such as maternal age, parity or type of attendance at which the risk of having a cesarean section increased significantly.

\section{Results}

During the study period, 11,026 singleton deliveries were reviewed. Of them, 1,828 were excluded due to incomplete data of either labor outcomes or maternal height. The remaining 9,198 were available for analysis. Of these, 5,201 (56.5\%) were nulliparous and 3997 (43.5\%) were multiporous women. In addition, most mothers (7,743; $84.2 \%$ ) attended the general antenatal clinic, taken care by rotating obstetricians while $1,455(15.8 \%)$ were taken care by one special obstetrician as a private service. The means \pm SD (range) of maternal age, gestational age at delivery, and birth weight were $28.02 \pm 6.02$ year (1344 years), $38.19 \pm 1.54$ weeks (34-43 weeks), and 2,983.7 $\pm 447.9 \mathrm{~g}(1,790-5,300 \mathrm{~g})$, respectively. The means $\pm \mathrm{SD}$ (range) maternal height was $155.4 \pm 6.2 \mathrm{~cm}(100-192 \mathrm{~cm})$, median (50 percentile) was $155.0 \mathrm{~cm}$. The percentile distribution of the maternal height at $5,10,20,50,80,90,95$ percentile were $145,148,150,155,160,163$ and $165 \mathrm{~cm}$, respectively.

In a total of 9,198, methods of delivery included normal vaginal delivery; $70.2 \%$, operative vaginal delivery; $7.5 \%$, and cesarean section; $22.3 \%$. The cesarean section rate due to CPD was $8.1 \%$ of all deliveries. Of interest, overall cesarean section rate in the mothers of nulliparity was slightly but significantly lower than that in the multiparous group ( 20.7 vs. $24.4 \% ; p<0.001$, odds ratio 0.92 ). Nevertheless, conversely, cesarean rate secondary to CPD was markedly higher among the nulliparous group (12.2 vs. $2.7 \% ; p<0.001$, odds ratio of 4.9 ).

The rates of cesarean section due to CPD with odds ratio in the groups categorized by maternal height and type of attendance, and parity are presented in Table 1 . In the group of short maternal height, using a cut-off value of $145 \mathrm{~cm}$, had a cesarean rate of $16.3 \%$ and odds ratio of 2.4. Note that the rate of cesarean section due to CPD declined from $16.3 \%$ at cut-off $145 \mathrm{~cm}$ to $11.4 \%$ at $150 \mathrm{~cm}$ and $9.6 \%$ at $155 \mathrm{~cm}$. Interestingly, the cesarean rate due to CPD in the private service was $13.6 \%$ with odds ratio of 2.1 . 


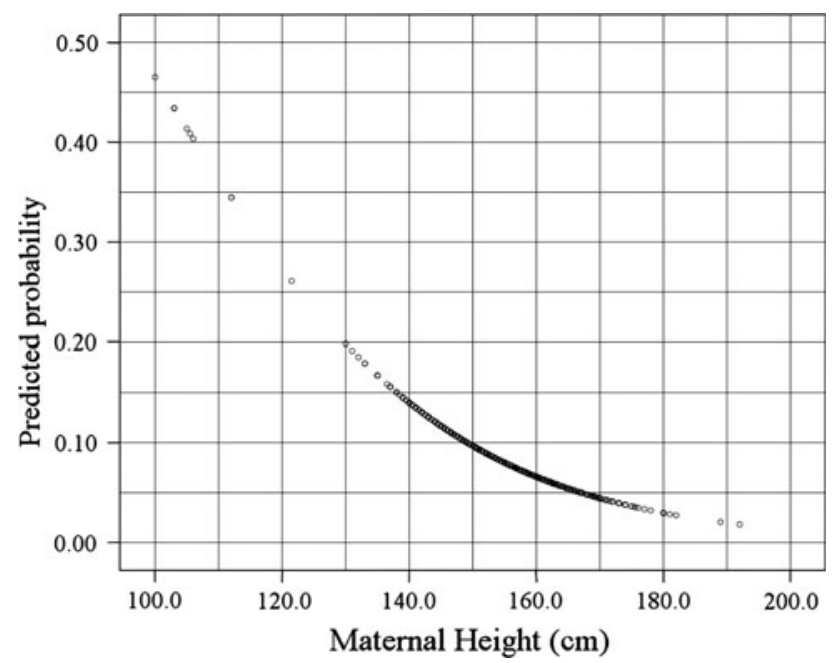

Fig. 1 Correlation between maternal height and predicted probability for cesarean section due to CPD regardless of other variables

When short stature was defined by a cut-off value of $145 \mathrm{~cm}$, the means \pm SD of maternal age of the short and normal mothers were not significantly different $(27.6 \pm 6.1$ vs. $28.0 \pm 6.0$ years, $p>0.05$ ) while the means \pm SD of birth weight in the short mothers was significantly lower than that in the normal height group $(2854 \pm 430$ vs. $2991 \pm 447 \mathrm{~g}, p<0.05)$.

In logistic regression analysis using maternal height as a single-independent variable, it was found that maternal height was significantly correlated with rate of cesarean section due to CPD $(p<0.0001)$. The equation of relationship is as follows (Fig. 1):

- Odds $=\exp (4.048-0.042 \times \mathrm{Ht})$; where, Ht is maternal height in $\mathrm{cm}$, odds represents a ratio of probability of cesarean section to probability of vaginal delivery.

Other variables which were found to be significant in multiple logistic regression analysis included maternal age, birth weight, parity, and private service. The equation is as follows:

- Odds $=\exp (-1.455-0.078 \times \mathrm{Ht}+0.085 \times$ age $+0.002 \times$ $\mathrm{BW}+0.566 \times$ private $+2.427 \times$ parity); where, $\mathrm{Ht}$ is maternal height in $\mathrm{cm}$, age represents maternal age in years, private is 1 if taken care in private service, parity is 1 for nuliparity).

\section{Discussion}

Though several studies have been published on this issue, our study was unique in terms of particular ethnic group and our findings should encourage all other groups around the world to evaluate the risk based on height distribution among their own population.
In our practice, we usually use a 145 -cm cut-off value for diagnosis of short stature, labeled as high risk, certainly different from that in the western since our mothers have a relatively small size, nevertheless this routine practice has never been evaluated for appropriateness. As seen in the results, despite the tendency to have relatively small infants, mothers with short stature were more likely to have a higher chance of obstructed labor. Based on our study, whereas other variables are not considered, a cut-off value of 145 , consisting of $5 \%$ of our obstetric population, is justified since the rate of CPD is about $16.3 \%$ and odds ratio of 2.4 while the overall cesarean rate was $8.1 \%$. Therefore, maternal height may be used as a simple tool to screen a risk factor for CPD and the need for highly specialized care. The logistic regression analysis has demonstrated a curve with a gradual decrease in the risk of cesarean section due to CPD with increasing maternal height. This relationship is a useful guide for counseling the couple in terms of clinical risk for cesarean section. However, in real practice, other variables other than maternal height are usually taken into account. Certainly a short mother with estimated low birth weight does not carry a high risk for CPD. Though a little more complicated, the equation derived from multiple logic regression analysis in this study may be helpful to quantify risk with more accuracy. Indeed, in the era of digital world, probability and odds calculation for cesarean section for CPD based on equation mentioned above are readily anywhere even in the rural area. Therefore, we suggest multiple logistic regression equation in risk calculation rather than maternal height alone.

Note that, we did not include mothers with gestational age of less than 34 weeks of gestation, since it is very rare in our practice to encounter CPD at such early gestation regardless of maternal height.

The study involved population in which the mothers are shorter than other groups ever studies even in Asian groups like Japanese [10]. Our data also suggest that a higher cesarean rate due to CPD should be expected for Thai population or probably Southern Asian mothers with shorter stature, even for those with relatively small babies. This information is important for appropriate counseling of the couple, especially in developing countries or even in developed countries with mixed ethnic groups. Information on higher risk for CPD is essential for good clinical practice. Higher precaution must be given for mothers with a higher risk in term of close attention or preparation of cesarean section or timely labor induction in daytime when more manpower and experienced physicians are available.

This study has demonstrated that maternal height was inversely correlated with a rate of cesarean delivery secondary to cephalopelvic disproportion. As already known, maternal height in our population is certainly shorter than that in the western population. Therefore, we first 
determined the percentile range of height distribution in our pregnant women, and then categorized them based on the height percentile groups. The height distribution in this study suggested that the height of $145 \mathrm{~cm}$ may be an appropriate cut-off for short stature in Thai population, instead of adopting a particular cut-off value as recommended in several previous studies which are in range of $150-160 \mathrm{~cm}[4,6,7,11]$.

Obviously, simply adopting a cut-off value of either 150 or 155 or $160 \mathrm{~cm}$ to define short stature in Thai or other ethnic groups in the same geographical area such as Laotian or Cambodian is inappropriate. For example, with definition of $155 \mathrm{~cm}$ as a cut-off value which is a fifth percentile value for German [8], $60 \%$ of our population, instead of $5 \%$ as suggested by several authors, would be classified as high risk for CPD and be likely to result in inconclusive results. That is an unacceptably great number for taking care of mothers at high risk.

In spite of the fact that the shorter in maternal height, the lower in birth weight, maternal height is inversely correlated with a higher risk of cesarean delivery due to cephalopelvic disproportion. The effect of maternal height on such cesarean rate seems to be continuous without definite cut-off point.

Based on our study and other previous studies in the western, the criterion for short stature should be definitely based on ethnic groups and must be categorized according to height percentile range rather than absolute height may allow comparison between different populations on the effect on labor outcome.

Certainly, birth weight is directly correlated to cesarean section rate due to CPD. In fact, infants born to mothers with normal height and CPD were heavier than those without CPD. Conversely, less heavy infants born to mothers with short stature might have been able to deliver normally. Nevertheless, after addition of birth weight in the logistic regression model, the correlation between CPD and maternal height remained high. Furthermore, mean birth weight in the group of short stature was less than that of the normal group. Therefore, it may be of value to combine maternal height and estimated fetal weight to assess the risk of CPD, though prenatally estimated birth weight may be not perfectly accurate. Likewise, nulliparity and private service significantly contributed to a higher risk for CPD and should also be taken into account.

In conclusion, mothers with short stature were independently correlated with a higher rate of CPD. Clinical points could be drawn from this study including (1) definition of short statue must be developed for particular geographic or ethnic groups. In Thai population, using $145 \mathrm{~cm}$ as a cut-off value, odds of CPD is 2.4; (2) probability of CPD may be estimated by maternal height as a single variable or multiple variables using logistic regression equations.

Acknowledgments We wish to thank the National Research University Project under Thailand's Office of the Higher Education Commission for financial support.

Conflict of interest No conflict of interest.

Open Access This article is distributed under the terms of the Creative Commons Attribution Noncommercial License which permits any noncommercial use, distribution, and reproduction in any medium, provided the original author(s) and source are credited.

\section{References}

1. Hogan MC, Foreman KJ, Naghavi M, Ahn SY, Wang M, Makela SM et al (2010) Maternal mortality for 181 countries, 1980-2008: a systematic analysis of progress towards Millennium Development Goal 5. Lancet 375(9726):1609-1623

2. Maternal anthropometry and pregnancy outcomes (1995) A WHO collaborative study. Bull World Health Organ 73(Suppl):1-98

3. Dujardin B, Van CR, Lambrechts T (1996) The value of maternal height as a risk factor of dystocia: a meta-analysis. Trop Med Int Health 1(4):510-521

4. Kara F, Yesildaglar N, Uygur D (2005) Maternal height as a risk factor for Caesarean section. Arch Gynecol Obstet 271(4): 336-337

5. Liselele HB, Boulvain M, Tshibangu KC, Meuris S (2000) Maternal height and external pelvimetry to predict cephalopelvic disproportion in nulliparous African women: a cohort study. BJOG 107(8):947-952

6. McGuinness BJ, Trivedi AN (1999) Maternal height as a risk factor for Caesarean section due to failure to progress in labour. Aust N Z J Obstet Gynaecol 39(2):152-154

7. Sheiner E, Levy A, Katz M, Mazor M (2005) Short stature-an independent risk factor for Cesarean delivery. Eur J Obstet Gynecol Reprod Biol 120(2):175-178

8. Bohlmann MK, Luedders DW, Beyer D, Kavallaris A, Baumann K, Diedrich K et al (2010) Nulliparous patients with small stature delivering at term have an increased risk of secondary cesarean section. Arch Gynecol Obstet 282(3):241-244

9. Sub-committe of Maternal and Child Health (2001) Diagnostic guideline for cesarean section due to cephalopelvic disproportion or failure to progress of labor. Obstet Gynecol Bull 10:17-22

10. Chan BC, Lao TT (2009) The impact of maternal height on intrapartum operative delivery: a reappraisal. J Obstet Gynaecol Res 35(2):307-314

11. Mahmood TA, Campbell DM, Wilson AW (1988) Maternal height, shoe size, and outcome of labour in white primigravidas: a prospective anthropometric study. BMJ 297(6647):515-517 